\title{
Sentinel lymph node in cervical cancer
}

\author{
Setare Akhavan ${ }^{1}$, Soheila Aminimoghaddam ${ }^{2 *}$, Maryam Rahmani ${ }^{3}$
}

Received: 23 August 2016

Published: 31 Aug 2017

\begin{abstract}
Background: Cervical cancer is the second most common type of cancer among women. Effective screening programs can help cancer detection in early phases and reduce death. Metastasis to lymph nodes is one of the most prognostic factors in patients who underwent surgery. Also, a positive result from pathology report alert oncologist as a cause of death. Sentinel lymph node biopsy has been widely studied and clinically used for many types of cancer.

Methods: Two techniques exist for detecting sentinel node in cervical cancer, which are Blue dye and gamma probe with radioactive isotope $(99 \mathrm{mTc})$. Moreover, lymphoscintigraphy has many advantages over the stain method. Detecting the sentinel node is performed via laparoscopy or laparotomy; former method is better and more accurate.

Results: Various researchers have focused on this method and its positive results; its superiority against full lymphadenectomy has been declared in previous studies. Moreover, the role of sentinel lymph nodes biopsy in cervical cancer is still being extensively studied. Sentinel lymph nodes (SLN) method has a higher accuracy level to detect metastasis.

Conclusion: Hence, it can be considered as a more appropriate alternative for pelvic lymph node dissection (PLND), which is a standard technique. Altering the method to a standard clinical method needs in-depth researches and studies.
\end{abstract}

Keywords: Biopsy, Sentinel lymph nodes, Cervical cancer, Blue dye

Copyright $\odot$ Iran University of Medical Sciences

Cite this article as: Akhavan S, Aminimoghaddam S, Rahmani M. Sentinel lymph node in cervical cancer. Med J Islam Repub Iran. 2017 (31 Aug);31:50. https://doi.org/10.14196/mjiri.31.50

\section{Introduction}

Invasive cervical cancer is the most common type of cancer among women after breast cancer in most regions of the world and 500000 new cases are detected every year (1).

Effective screening methods can help diagnose cervical cancer in its early stages and reduce morbidity and mortality. Otherwise, patients should tolerate the side effects of the treatment for a long time. Thus, timely diagnosis to prevent the progress of the cancer and reduce treatment side effects is of prime importance. Metastasis to lymph nodes is a common pathway during the natural course of cervical cancer progress, and metastasis to lymph nodes with tumoral involvement is the most important cause of deterioration and even death among those patients who

Corresponding author: Dr Soheila Aminimoghaddam,dr_aminimoghaddam@yahoo.com

1. Department of Obstetrics and Gynecology, Medical School, Tehran University of Medical Sciences, Tehran, Iran.

2. Department of Obstetrics and Gynecology, Medical School, Iran University of Medical Sciences, Tehran, Iran.

3. Department of Radiology, Medical School, Tehran University of Medical Sciences, Tehran, Iran. have undergone surgery. If the PLN test is positive, the patient's 5- year survival rate reduces from $92 \%$ to $64 \%$. Sentinel lymph node (SLN) is the first lymph for drainage of lymph from the initial tumors and it indicates the progression of cancer in the lymphatic system $(2,3)$. Thus, if the sentinel lymph node is free of any cancer, the remaining lymph nodes in the vicinity of tumor should also be free of any disease, and there will be no need for full lymphadenectomy. The possibility of lymph nodes metastasis in cervical cancer in the early stage is only $27 \%$. As a result, it is possible that at least 3 out of each four patients undergo unnecessary PLND, and consequently, suffer from its side effects and experience less survival benefit (4). SLN method is more accurate in detecting metastasis;

$\uparrow$ What is "already known" in this topic:

Sentinel node biopsy was used in gynecologic cancer especially in vulvar cancer. However, this procedure is applicable in cervical carcinoma. The literature shows that using the blue dye besides using TC99 has an advantage of detecting the sentinel node with higher accuracy. This shows that lymphadenectomy could be omitted when sentinel node is negative.

\section{$\rightarrow$ What this article adds:}

Laparoscopy for detecting the sentinel node at fertility sparing surgery in early stages of cervical cancer with trachelectomy is a better procedure. Moreover, the results show that IHC can be used as a supplementary approach for detecting sentinel node with higher accuracy. 
thus, it can be considered as a more appropriate alternative rather than PLND.

SLN is a standard diagnostic method, which is applicable in patients with breast cancer, but it can also be used in patients with cervical cancer. On the other hand, the probability of lymph nodes metastasis among patients with tumors smaller than or equal to $2 \mathrm{~cm}$ ranges from 0 to $16 \%$, while this possibility among those patients who have IB stage ranges from 15 to $31 \%$. As a result, a significant number of patients with negative sentinel lymph node test would not require lymphadenectomy, so the side effects such as lymphedema (10-15\%), formation of lymphocytes (more than 20\%), neurovascular damages, vascular thromboembolism, and infection are reduced (5-8).

Another advantage of this method is the higher levels of diagnosing metastasis to lymph nodes through staging (up to $25 \%$ rise in diagnosing metastasis and identifying micrometastasis) $(9,10)$. Furthermore, early diagnosis of cancer through such methods can prevent the simultaneous use of radical surgery and radiotherapy, which is highly important in preserving fertility among young women (11).

\section{Background}

The first studies on lymph vessels were conducted by Sappey about 100 years ago. Sherman et al. announced the finding of a regular vascular pattern in 1953. In 1977, Kabanas diagnosed the first lymph node in a patient with genital tract malignancy and used the term sentinel lymph node. Kabanas initiated a method, which was later used by other scientists to diagnose and treat other types of cancer (12).

In 1992, Morton et al. published their first findings on lymph mapping of the melanoma by injecting dye in the region and diagnosing sentinel lymph nodes in 194 cases. The percentage of metastasis among the lymph nodes was $21 \%$, while this percentage was reported to be much smaller in other lymph nodes (2 lymph nodes of 3000 nodes studied) (13). Lymph node mapping of melanoma with injection of radioisotope was described for the first time in 1993 (14).

\section{Methods}

\section{Dye stain}

In this method, isosulfan blue, patent purple-blue, and methylene blue are usually used. The blue dye is injected around the tumor and absorbed by the lymphatic system 5 to 15 minutes later, and dye can stay there for 60 minutes. Injection of high levels of dye inside the tumor or the vessels can demonstrate high levels of background signal, which would result in low diagnostic accuracy (15). Another problem with this method occurred in large tumors with necrosis in the center. This can usually result in infiltration of dye into the vagina through the cervical canal. familiarity with residual stroma, using a long spinal needle and controlling the infiltration of dye into the vagina, can help increase the precision $(16,17)$. This dye has weak connections with plasma proteins and it is usually easily disposed of through biliary ducts. Side effects such as changing the color of urine into blue, allergic reactions, and sometimes severe reactions due to the mixed dye with local anesthetic agent are also reported (3).

\section{Injection of radioactive isotopes}

For this purpose, $99 \mathrm{mTc}$-sulfur colloid is used in the US, while 99mtc-nanocolloid is utilized in Europe. Dynamic photography starts after the injection and usually lasts for 20 to 30 minutes to illustrate the lymphatic drainage and diagnose sentinel lymph node. A gamma probe is used to identify dynamic signals. Due to the slow and smooth flowing of the fluid, even small lymph nodes are detectable in scanning. Hot nodes were diagnosed compared with their brighter background (12).

Dose and type of the radioactive material injected, the time elapsed between the injection and surgery, and the type of the gamma probe utilized influence the precision of the scan and the ratio of sentinel lymph nodes to background lymph nodes. This ratio usually ranges from 10:1 to $25: 1$. The sentinel nodes make up at least $20 \%$ of the hot nodes counted in pelvis (17).

The method, known as lymphoscintigraphy, has many advantages over the stain method. The location of the lymph nodes is found more accurately and the risk of bleeding during the surgery reduces. It may also be possible to remove lymph nodes through a small incision in abdomen. On the other hand, this technique is more expensive and requires more time and a nuclear medicine team with security protocols for operation. In addition, blue dye will be more applicable when sentinel lymph node is located in parametrium because counting with gamma probe is prone to errors due to the influence of vicinity to cervix and overlapping with it. The possibility of diagnosing sentinel lymph nodes in the cervical carcinoma has been reported among 800 patients, for $81 \%$ of whom dye stain method was used (18). Another method has also been proposed, known as the combined method, which seems to be the most sensitive diagnostic method for evaluation of lymph nodes afflicted by cervical cancer in the early phases as compared with methods such as positron emission tomography and magnetic resonance photography (19). Laparoscopy is a safe and accurate method to diagnose the sentinel lymph nodes using stain and radioisotope in the combined method $(20,21)$. Pooled SLN detection rate is $96.1 \%$, which is greater than the detection rate in laparotomy $(90.2 \%)$. The comparison of sensitivity between laparoscopy and laparotomy in detecting sentinel lymph node shows that the former has higher sensitivity rate $(89.8 \%$ vs. $86.3 \%)$.

\section{Discussion}

Although various studies have been conducted on the efficacy of this method to treat patients with cervical cancer, it is highly important to keep in mind some tips before we introduce this critical clinical routing technique. Firstly, keeping in mind the fact that utilizing merely the blue stain will result in low diagnostic rate, it is necessary to use a radioactive detector to achieve higher diagnostic rates. Furthermore, factors such as type, volume, or length of using the radioactive material should be determined before a standard protocol. 
Second, pelvic lymphadenectomy surgery requires the presence of pathologists and quick pathological diagnostic methods. Errors in the negative state of pathology are among other risks of this technique. Thus, using immunohistochemical techniques among other techniques, especially in large tumors or advanced cases would result in massive lymphatic damages (22). Diagnosis of sentinel lymph nodes in parametrium might be difficult (23). Furthermore, it is not always possible to diagnose the sentinel lymph nodes in both sides. Under such conditions, full lymphadenectomy should be conducted on the side, where diagnosis is not possible (24). It is worth noting that $15 \%$ to $43 \%$ of the lymph nodes cannot be diagnosed even with immunohistochemical and PCR techniques, which oblige researchers to use new methods to detect micrometastasis (25-27).

The following algorithm proposed by Vicus and Covens can be used as a guide in measuring and evaluating sentinel lymph nodes. Injection of technetium in all 4 quadrants of the cervix was conducted through submucosal tunnel. If the sentinel lymph nodes are diagnosed only on the one side, full lymphadenectomy has to be performed on the other side (28). Instead of primary hysterectomy, a systematic assessment of all lymph nodes can be done in the first step, which is followed by a radical hysterectomy (29). It is proposed that sentinel lymph node is performed rather than total lymphadenectomy before trachelectomy in young patients with cervical cancer who have a desire for pregnancy.

Traditionally, biopsy of the sentinel lymph nodes was conducted as a substitute for total lymphadenectomy of the pelvis to determine local and regional lymph node tumor of the cervix in patients with Stage 1 cervical cancer with tumor size of $2 \mathrm{~cm}$. This method can be used in each histologic type such as squamous, adenosquamous, and adenocarcinoma. However, according to the midline position of the cervix, the lymphatic drainage of cervix is complicated. In brief, using the combined method, dye stain and radioisotope, is the best reliable method to diagnose metastasis in lymph nodes.

Mapping sentinel lymph nodes is popular among gynecologist- oncologists, which lead to having fewer side effects in patients who suffer from cervical cancer. Thus, more researches are required to confirm the total removal of lymph nodes in patients with sentinel lymph nodes positive. The presence of an effective team (composed of an expert gynecologist- oncologist and nuclear medicine team) is an important factor to have a successful surgery with an acceptable diagnostic power, less invasive operations, and better clinical management.

\section{Conclusion}

Sentinel lymph node should be considered in early stages of cervical cancer without metastatic disease, especially in patients with a desire for pregnancy. Laparoscopy, combination of radio nucleoid with blue dye, IHC, and HE have higher SLN detection rate.
Conflict of Interests

The authors declare that they have no competing interests.

\section{References}

1. Parkin DM, Bray F, Ferlay J, Pisani P. Global cancer statistics, 2002. Ca Cancer J Clin. 2005;55(2):74108.

2. Aminimoghaddam S, Mahmoudzadeh F, Maghsoudnia A. Cervical cancer along with unknown cirrhosis: a misdiagnosed case. JRI. 2015;16(3):174.

3. Levenback C. Update on sentinel lymph node biopsy in gynecologic cancers. Gynecol Oncol. 2008;111(2):S42-S43.

4. Casper T, Rovers MM, de Graaf C, Zusterzeel PLM, Bekkers RLM. The sentinel node procedure in early stage cervical cancer, taking the next step; a diagnostic review. Gynecol Oncol. 2015;139(3): 559-567.

5. Acharya BC, Jihong L. Sentinel lymph node detection in patients with early cervical cancer. J Nepal Med Assoc. 2009;48(176):287-291.

6. Franchi M, Ghezzi F, Riva C, Miglierina M, Buttarelli M, Bolis P. Postoperative complications after pelvic lymphadenectomy for the surgical staging of endometrial cancer. J Surg Oncol. 2001;78(4):232237.

7. Levenback C, Coleman RL, Burkeetal TW. Lympathic mapping and sentinel node identification in patients with cervix cancer undergoing radical hysterectomy and pelvic lymphadenectomy. J Clin Oncol 2002;20(30):688-693.

8. Matsuura Y, Kawagoe T, Toki N, Tanaka M, Kashimura M. Longstanding complications after treatment for cancer of the uterine cervix - clinical significance of medical examination at 5 years after treatment. Int J Gynecol Cancer. 2006;16(1):294-297.

9. Euscher ED, Malpica A, Atkinson EN, Levenback CF, Frumovitz M, Deavers MT. Ultra staging improves detection of metastases in sentinel lymph nodes of uterine cervix squamous cell carcinoma. Am J Surg Pathol. 2008;32(9):1336-1343.

10. Obnisbi S, Lomnes SJ, Laurence RG, Gogbasbian A, Mariant G, Frangioni JV. Organic alternatives to quantum dots for intraoperative near-infrared fluorescent sentinel lymph node mapping. MOL Imaging. 2005;4(3):172-181.

11. Du XL, Sheng XG, Jiang T, Li QS, Yu H, Pan CX, Lu CH, Wang C, Song QQ. Sentinel lymph node biopsy as guidance for radical trachelectomy in young patients with early stage cervical cancer. BMC Cancer. 2011;11.

12. Cibula D, Oonk MH, Abu-Rustum NR. Sentinel lymph node biopsy in the manage- ment of gynecologic cancer. Curr Opin Obstet Gynecol. 2015;27(1):66-72.

13. Ruscito I, Gasparri ML, Braicu EI, Bellati F, Raio L, Sehouli J, et al. Sentinel node mapping in cervical and endometrial cancer: indocyanine green versus other con- ventional dyes - a meta-analysis. Ann Surg Oncol. 2016 Oct 1;23(11):3749-56.

14. Ghaoomi M, Aminimoghaddam S, Safari H, Mahmoudzadeh A. Awareness and practice of cervical cancer and Pap smear testing in a teaching hospital in Tehran. TUMJ. 2016 Jun 15;74(3):183-9.

15. El-Ghobashy AE, Saidi SA. Sentinel lymph node sampling in gynaecological cancers: techniques and clinical applications. EUR J Surg Oncol. 2009; 35(7):675-685.

16. Angioli R., Palaia I., Cipriani C., Muzii L., Calcagno M., Gullotta G. Panici P.B. Role of sentinel lymph node biopsy procedure in cervical cancer: a critical point of view. Gynecol Oncol. 2005;96(2):504-509.

17. Mariani G, Gipponi M, Moresco L, et al. Radioguided sentinel lymph node biopsy in malignant cutaneous melanoma. JNM 2002;43(6):811-827.

18. Aminimogha S, Shadman L, Hashemi RS, Mohammadi Z, Mahmoudzad F. sentinel lymph node detection using methylene blue in Iranian patients with early stage cervical cancer. J Applied Sci; 2015 Mar; 15:582-7.

19. Aminimoghaddam S, Mahmoudzadeh F, Maghsoudnia A, Ghaemmaghami F. Intraoperative visualization, frozen section, and permanent pathology in endometrial cancer. TUMJ. 2014 Dec 15;72(9):61722 .

20. Barranger E, Grahek D, Cortez A, Talbot JN, Uzan S, Darai E. Laparoscopic sentinel lymph node procedure using a combination of patent blue and radioisotope in women with cervical carcinoma. Cancer. 2003;97(12):3003-3009.

21. Mart'inez-Palones JM, Gil-Moreno A, P'erez-Benavente MA, Roca 
I, Xercavins J. Intraoperative sentinel node identification in early stage cervical cancer using a combination of radiolabeled albumin injection and isosulfan blue dye injection. Gynecol Oncol. 2004;92(3):845-850.

22. D'1az-Feijoo B, Gil-Moreno A, P'erez-Benavente MA, Morch' on S, Mart'inez-Palones JM, Xercavins J. Sentinel lymph node identification and radical hysterectomy with lymphadenectomy in early stage cervical cancer: laparoscopy versus laparotomy. J Minim Invas Gyn. 2008;15(5):531-537.

23. Covens A, Rosen B, Murphyetal J. How importantis removal of the parametrium at surgery for carcinoma of the cervix? Gynecol Oncol. 2002;48(1):145-149.

24. Darlin L., Persson J., Bossmar T., Lindahl B., Kannist P., Måsbäck A. and Borgfeldt $C$. The sentinel node concept in early cervical cancer performs well in tumors smaller than $2 \mathrm{~cm}$. Gynecol Oncol. 2010;117(2):266-269.

1. Lentz SE, Muderspach LI, Felix JC, Ye W, Groshen S, Amezcua CA. Identification of micrometastases in histologically negative lymph nodes of early-stage cervical cancer patients. Obstet Gynecol. 2004;103(6):1204-1210.

2. Wang HY, Sun JM, Lu HF, et al. Micrometastases detected by cytokeratin 19 expression in sentinel lymph nodes of patients with earlystage cervical cancer. Int J Gynecol Cancer. 16(2):643-648.

3. Dara"1 E, Rouzier R, Ballester M, Barranger E, Coutant C. Sentinel lymph node biopsy in gynaecological cancers: the importance of micrometastases in cervical cancer. SURG ONCOL. 17(3):227-235.

4. Vicus D, Covens A. Role of sentinel lymph node biopsy in cervical cancer: pro. Int J Gynecol Cancer. 2010;20(11):S34-S36.

5. Bats A.S., Buénerd A., Querleu D., Leblanc E., Daraï E., Morice P., Marret H., Gillaizeau F., Mathevet P. and Lécuru F., Diagnostic value of intraoperative examination of sentinel lymph node in early cervical cancer: a prospective, multicenter study. Gynecol Oncol. 2011;123(2):230-235 